\title{
Effect of Pronation and Postural Changes on Non-intubated Patients with Respiratory Failure Due to Covid-19 Interstitial Pneumonia
}

\section{Lorenzo Porta $^{1,2 *}$, Giulio Cassano ${ }^{1,2}$, Silvia Gheda ${ }^{1,2}$, Adriano Basile $^{1}$ and Andrea Bellone ${ }^{1}$}

${ }^{1}$ Emergency Medicine Department, Azienda Socio-Sanitaria Territoriale Grande Ospedale Metropolitano Niguarda, Milano, Italy

${ }^{2}$ University of Milan-Bicocca, School of Medicine and Surgery, Monza, Italy

*Corresponding Author: Lorenzo Porta, Emergency Medicine Department, Azienda

Socio-Sanitaria Territoriale Grande Ospedale Metropolitano Niguarda, Milano, Italy.
Received: October 05, 2021

Published: November 17, 2021

(C) All rights are reserved by Lorenzo Porta., et al.

\section{Abstract}

Background: There is still paucity of data regarding pronation on non-intubated patients with respiratory failure due to Severe Acute Respiratory Syndrome Coronavirus-2 (SARS-CoV-2).

Objective: To evaluate the effects of prone and lateral positioning on acute respiratory failure (ARF) due to SARS-CoV-2 interstitial pneumonia.

Methods: We retrospectively analyzed data of all patients admitted to our medical ward from the $1^{\text {st }}$ of March to the $15^{\text {th }}$ of $A p r i l$ 2020 with acute respiratory failure due to SARS-CoV-2 pneumonia. We compared patients mobilized in either lateral or prone positions with those mobilized just in sitting position. We recorded data on postural changes and all possible confounding variables. We assessed the relationship between postural changes and necessity of endotracheal intubation (ETI), obtaining crude and adjusted Odds Ratios (ORs), using conditional logistic regression analysis. Furthermore, we analyzed mortality for DNI (do not intubate) and actual intubation for non DNI, respectively.

Results: Our population of 98 patients had a male/female ratio of 3.25, a mean age of 65,6 years, a mean P/F of 183,3 and a median APACHE II score of 9. Crude and adjusted ORs with Confidence Interval (CI) for the need of ETI were 0,239 (CI95, 0,102-0,556, p 0,001) and 0,045 (CI95, 0,008-0,244, p < 0,001). Crude and adjusted ORs for ETI in the non-DNI population were 0,137 (CI95, 0,044-0,422, p 0,001) and 0,076 (CI95, 0,014-0,404, p 0,002), respectively. The OR for mortality in the DNI subgroup did not achieve statistical significance, even after adjustment. Compared to the sitting position, prone and lateral positions were associated with higher improvement in mean $\mathrm{SpO}_{2}(+5.25 \%$ and $+4.50 \%$, respectively), with a partial benefit persistent after resupination $(+3.68 \%$ and $+1.87 \%$, respectively) .

Conclusions: Our study claims that the systematical application of prone and/or lateral positions can reduce ETI in SARS-CoV-2 patients with mild to severe ARF, due to a beneficial effect on $\mathrm{SpO}_{2}$ and, thus, tissue hypoxia.

Keyword: Prone Positioning; SARS-CoV-2; Interstitial Pneumonia; Prone Ventilation; Acute Respiratory Failure; Non-intubated Patients

\section{Introduction}

Since its identification at the end of 2019, the novel coronavirus (SARS-CoV-2) rapidly spread around the world, exponentially increasing the number of the affected. Despite a majority of asymp- tomatic patients, SARS-CoV-2 can cause mild to severe infections, with pneumonia as its most common manifestation [1,2]. SARSCoV-2 pneumonia manifests with chest CT imaging abnormalities with a rapid evolution from focal unilateral to diffuse bilateral 
ground-glass opacities, that can either progress to or co-exist with consolidations within 1-3 weeks [2,3]. Three relatively large-scale case studies have thoroughly demonstrated the clinical features of patients with SARS-CoV-2 pneumonia in Wuhan [4-6]. The disease is characterized initially by fever, cough and dyspnea, but, with its progression, can evolve in acute respiratory failure (ARF), acute respiratory distress syndrome (ARDS), shock and multiorgan dysfunction. Of the aforementioned complications, ARDS is one of the most frequent. In fact, in Wang., et al. study, ARDS developed in 20\% of their patients, whereas, in Wu., et al. study in 41\% [4,7]. Due to both its prevalence and association with characteristics typical of western countries population (age greater than 65 years, diabetes mellitus and hypertension), SARS-CoV-2 ARDS can severely affect the course and outcome of the disease.

Prone positioning (PP) has been demonstrated successful in the management of ARDS [8] and anecdotical observations have suggested its possible application in SARS-CoV-2 patients admitted to the intensive care units (ICU) [9]. Moreover, the Surviving Sepsis Campaign panel recently recommended that "mechanically ventilated patients with COVID-19 should be managed similarly to other patients with acute respiratory failure in the ICU suggesting prone ventilation for 12-16 h, over no prone ventilation for mechanically ventilated adults with COVID-19 and moderate to severe ARDS" [10]. The mechanisms by which prone position improves gas exchange include alveolar recruitment, redistribution of ventilation towards dorsal areas, homogenization of tidal volume distribution and possible improved postural drainage of secretions [12,13]. Besides, it has been suggested that postural drainage could also reduce ventilator-associated pneumonia, although it could theoretically spread organisms and inflammatory mediators within lung tissue, leading to an increased organ damage [14,15]. Likewise, homogenization of tidal volume distribution might reduce tissue stress/strain and consequently diminish the injurious effects of mechanical ventilation [16-19]. Indeed, several trials have evidenced a beneficial effect of pronation on $\mathrm{PaO}_{2}$, which lingers for hours after resupination [20-21]. Furthermore, recent studies have demonstrated a mortality benefit from early, long-lasting PP ventilation cycles in patients with severe ARDS [22-28]. These aforementioned benefits should also apply to non-intubated patients, in whom PP improve oxygenation, thus, possibly delaying or even avoiding endotracheal intubation (ETI). In fact, even though reports of the application of PP in spontaneously breathing, non-intubated adult patients are limited to few case reports [29,30], it has been supported by Scaravillli., et al. in a retrospective study that pronation of awake, spontaneously breathing, non-intubated patients with hypoxemic ARF was feasible, safe, and associated with a significant benefit on oxygenation [31]. Thus, there is a strong rationale to apply pronation in the management of non-intubated patients in medical wards (MWs), as a strategy to improve oxygenation and prevent ETI, thus, relieving the burden on ICUs.

In fact, due to the exponential increase of the number of the infected, the demand for ETI and mechanical ventilation escalated, imposing more selective admission criteria to the ICU, not able to fulfill the request [32]. With this in mind, we decided to assess the feasibility and effectiveness of early cyclic periods of postural changes (prone and lateral) on SARS-CoV2 patients in MWs, by conducting a retrospective cohort study, including all patients admitted to our ward with acute respiratory failure due to SARSCoV2 interstitial pneumonia, with the typical radiographic pattern of ground-glass opacities and bilateral patchy shadowing.

\section{Materials and Methods}

We performed a retrospective cohort study by collecting the medical records of all patients admitted to the Emergency ward of Ospedale Maggiore Niguarda from the $1^{\text {st }}$ of March to the $15^{\text {th }}$ of April 2020. We screened their data for the following inclusion criteria:

- $\quad$ ARF on admission, defined as arterial partial pressure to inspired fraction of oxygen ratio $\left(\mathrm{PaO}_{2} / \mathrm{FIO}_{2}\right)$ lower than $300 \mathrm{mmHg}$, and need of oxygen or ventilation support to achieve a peripheral capillary oxygen saturation $\left(\mathrm{SpO}_{2}\right)$ higher than $90 \%$.

- Confirmation of SARS-CoV-2 pneumonia with a positive swab at admission and typical CT chest pattern of ground-glass opacities and bilateral patchy shadowing.

We excluded all SARS-CoV-2 patients admitted with a concomitant diagnosis (ie, bowel obstruction, syncope, stroke).

We proposed mobilization in lateral or prone decubitus to all patients that met the inclusion criteria. We compared patients who accepted to be mobilized in prone and lateral position with those who categorically refused this treatment and thus were mobilized just in sitting position. All patients were mobilized twice a day, during the morning and afternoon shifts, either in sitting, lateral or prone positions. 
We recorded all demographic data (i.e., gender, age), comorbidities, diagnosis and severity scores (Charlson Comorbidity Index, Acute Physiology and Chronic Health Evaluation II score [APACHE II] and Pneumonia Severity Index [PSI]) at admission, CT scans, SARS-CoV2 test results, blood exams during the hospitalization, pharmacological therapies administered, length of hospitalization, incidence of endotracheal intubation, mortality and all data on postural changes (lateral, prone and sitting decubitus). To evaluate the clinical effects of postural change, we collected data on vital signs, the overall duration of the mobilization, the type of respiratory support used (i.e., oxygen supply mask, high-flow nasal cannulas [HFNC], helmet continuous positive airway pressure [CPAP], non-invasive ventilation [NIV] mask), the maximum $\mathrm{SpO}_{2}$ reached prior, during and after every postural change and possible complications (i.e., displacement of indwelling catheters, facial edema, second-degree pressure sores or higher, pressure neuropathies, compression of nerves and retinal vessels, vomiting, and intolerance to the maneuver).

We prescribed to all patients a therapy for SARS-CoV-2 interstitial pneumonia composed of lopinavir/ritonavir, hydroxychloroquine, azithromycin, nutritional support (vitamins, amino acids, proteins and electrolytes supplements). This therapy was progressively changed according to the published international evidences, in both groups at the same time.

The primary aim of our research was to assess the effect of prone and lateral positions on the indication for ETI, defined as the presence of either one or more major criteria or at least two minor criteria (Figure 1), as previously applied by other studies (3335). Furthermore, we analyzed the relationship between postural changes and either mortality for DNI or actual intubation for non DNI, respectively. Patient's consent was not required for this study due to its observational retrospective nature, the encryption of all personal identifiers and the anonymous analysis.

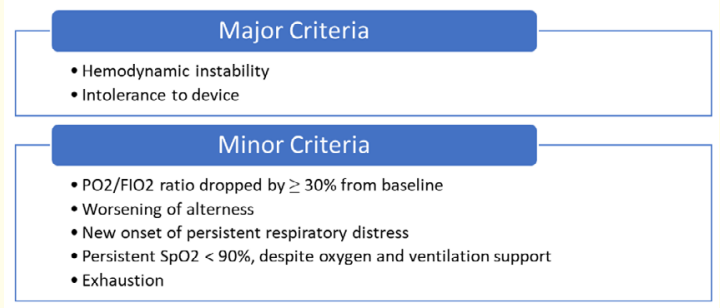

Figure 1: ETI Criteria.

\section{Statistical analyses}

Participants' baseline characteristics were presented as frequencies with percentages (categorical variables), median with interquartile range (quantitative ordinal variables) and mean with standard deviations (quantitative continuous variables). Normally distributed variables were compared by student's T-test, and nonnormally distributed variables were compared by Mann-Whitney $\mathrm{U}$ tests. A two-tailed P-value of less than 0.05 was considered statistically significant. We calculated the crude odds ratios and then adjusted for 15 individual covariates by logistic regression. We adjusted for the following variables: gender, age, BMI, presence of diabetes, hypertension, cardiovascular, respiratory or oncological disease, immunodepression (defined as the chronic use of immunosuppressants, such as calcineurin Inhibitors, antiproliferative agents, mTOR inhibitors or steroids), day of presentation to the emergency department, APACHE II, necessity of PEEP or $\mathrm{FIO}_{2}$ $>50 \%$ at admission, DNI or Tocilizumab prescription during the hospitalization. We took into consideration for the analysis the covariates that in literature have been associated with our outcomes and with the worsening of the disease. All analyses were carried out using IBM SPSS Statistics 25.0, and data were reported in accordance with STROBE guidelines.

\section{Results}

From a total of 178 patients admitted to our Emergency ward from the $1^{\text {st }}$ of March to the $15^{\text {th }}$ of April 2020, we identified 98 patients that met the inclusion criteria (Figure 2).

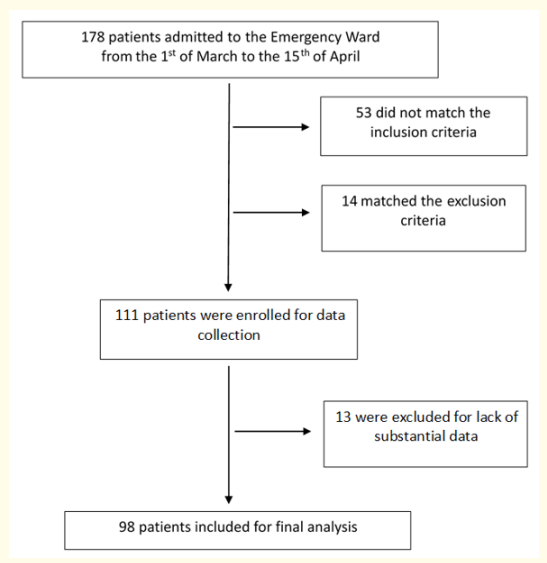

Figure 2: Inclusion Chart. 
The baseline characteristics of our population were shown in table 1. Our population had a mean age of 65 years old; it was composed for the majority by males (76.5\%), with hypertension, diabetes and obesity as the most represented concomitant diseases (49\%, 20\%, 31\%, respectively). At admission, the median PSI and Apache II were 81 and 9; the mean P/F was 183,3. To achieve a $\mathrm{SpO}_{2}$ greater than $90 \%, 70 \%$ of patients needed $\mathrm{FIO}_{2}$ greater than $>50 \%$, while $25 \%$ needed the addition of a Positive End Expiratory Pressure (PEEP). During the hospitalization, "do not intubate" (DNI) was applied to one third of the population, $27 \%$ of the population received Tocilizumab in addition to the standard treatment.
$64 \%$ of the population needed an increase of the $\mathrm{FIO}_{2}$ and $76,5 \%$ needed an increase in the ventilation support, defined as either a switch to another ventilation device or an increase in the PEEP value (ranging from 5 to $12.5 \mathrm{mmHg}$ ). During the hospitalization, our patients were sedated with low-dose morphine (20 to $40 \mathrm{mg}$ / die) to reduce the respiratory drive (RASS ranging from -1 to 3 ). Patients labeled as DNI were usually older (mean age 78.2 years old), with higher Charlson Comorbidity Index, APACHE II and PSI $(5,106,12.5$, respectively) and degree of concomitant diseases (13.3\% suffered from neoplastic disease, 43\% from cardiovascular disease and $30 \%$ from pulmonary disease).

\begin{tabular}{|c|c|c|}
\hline & \multicolumn{2}{|c|}{ Collective Population (98 patients) } \\
\hline & Mean with Standard Deviation & Range \\
\hline \multicolumn{3}{|c|}{ Demographics } \\
\hline Male sex $(\%)$ & $75(76,5 \%)$ & \\
\hline Age mean (year) & $65,6(12,7)$ & $27-90$ \\
\hline BMI (kg/m^2) & $28,4(5,2)$ & $20,8-45,8$ \\
\hline \multicolumn{3}{|c|}{ Co-pathologies } \\
\hline D Charlson Comorbidity index & $3(2-5)$ & $0-13$ \\
\hline${ }^{*}$ Hypertension (\%) & $48(49 \%)$ & \\
\hline Diabetes (\%) & $20(20,4 \%)$ & \\
\hline Obesity (\%) & $30(30,6 \%)$ & \\
\hline F Cardiovascular Disease (\%) & $18(18,4 \%)$ & \\
\hline * Respiratory Disease (\%) & $16(16,3 \%)$ & \\
\hline Oncological Disease (\%) & $6(6,1 \%)$ & \\
\hline${ }^{\ddagger}$ Immunodepression (\%) & $6(6,1 \%)$ & \\
\hline \multicolumn{3}{|c|}{ Characteristics at admission } \\
\hline${ }^{\delta}$ Day of presentation since the start of symptoms & $7(4,75-10)$ & $0-16$ \\
\hline${ }^{\delta} \mathrm{RR}$ & $24(21-30)$ & $14-42$ \\
\hline b PSI & $81(64-101,25)$ & $27-170$ \\
\hline${ }^{5}$ APACHE 2 & $9(6-13)$ & $2-22$ \\
\hline $\mathrm{pH}$ & $7,46(0,05)$ & $7,23-7,57$ \\
\hline pCO2 (mmHg) & $32,1(4,2)$ & $23-45$ \\
\hline $\mathrm{P} / \mathrm{F}(\mathrm{mmHg})$ & $183,3(78,7)$ & $40-298,6$ \\
\hline Sat02 (\%)5 & $91,4(4,6)$ & $66-99$ \\
\hline $\mathrm{HcO3}-(\mathrm{mmol} / \mathrm{l})$ & $22,7(3,3)$ & $15-38$ \\
\hline Lactate $(\mathrm{mEq} / \mathrm{l})$ & $1,77(1,58)$ & $0,3-9,8$ \\
\hline${ }^{\ddagger}$ Necessity of $\mathrm{FIO}_{2}>50 \%(\%)$ & $71(72,4 \%)$ & \\
\hline${ }^{*}$ Necessity of PEEP to achieve $\mathrm{SpO}_{2}>90 \%(\%)$ & $25(25,5 \%)$ & \\
\hline \#NI (\%) & $30(30,6 \%)$ & \\
\hline \multicolumn{3}{|c|}{ During the hospitalization } \\
\hline * Tocilizumab (\%) & $26(26,5 \%)$ & \\
\hline Necessity of increasing the $\mathrm{FIO}_{2}(\%)$ & $63(64,3 \%)$ & \\
\hline
\end{tabular}


Effect of Pronation and Postural Changes on Non-intubated Patients with Respiratory Failure Due to Covid-19 Interstitial Pneumonia

\begin{tabular}{|l|c|c|}
\hline${ }^{\ddagger}$ Necessity of increasing the ventilation support (\%) & $75(76,5 \%)$ & \multicolumn{2}{|l|}{} \\
\hline Outcome & $45(45,9 \%)$ & \\
\hline${ }^{\ddagger}$ Necessity of intubation (\%) & $25(36,8 \%)$ & \\
\hline${ }^{\ddagger}$ Intubation for non DNI (\%) & $19(63,3 \%)$ & \\
\hline${ }^{\ddagger}$ Mortality for DNI (\%) & \\
\hline
\end{tabular}

Table 1: Characteristics of the collective population.

Legend: All quantitative continuous data were reported as mean (standard deviations), quantitative ordinal variables (ర) were reported with median (interquartile range); all categorical variables ( $\ddagger$ ) were reported with frequencies (percentage). APACHE II (Acute Physiol-

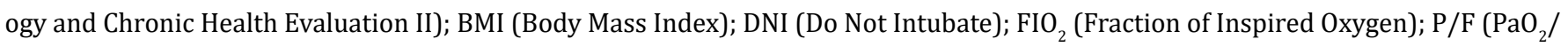
$\mathrm{FIO}_{2}$ ); PEEP (Positive End-Expiratory Pressure); PSI (Pneumonia Severity Index); $\mathrm{SpO}_{2}$ (peripheral capillary oxygen saturation).

In table 2 we displayed a significant difference between the two groups for immunodepression, day of presentation since the start of symptoms, admission respiratory rate, APACHE II, pH, necessity of $\mathrm{FIO}_{2}>50 \%$, prescription of Tocilizumab and the necessity of increasing the $\mathrm{FIO}_{2}$ during the hospitalization. In fact, patients in the lateral/prone positions group appeared to be more immune depressed, with longer time from the onset of symptoms to ad- mission, higher respiratory rate, APACHE II and necessity of $\mathrm{FIO}_{2}$ $>50 \%$ at admission. Compared to patients mobilized just in sitting position, a higher percentage of patients mobilized in prone or later positions were treated with Tocilizumab and were subjected to slightly higher degree of sedation, especially during prone positioning.

\begin{tabular}{|c|c|c|c|}
\hline & $\begin{array}{c}\text { Prone/Lateral Position } \\
\text { Group }(n=53)\end{array}$ & $\begin{array}{l}\text { Sitting Position } \\
\text { Group }(\mathrm{N}=45)\end{array}$ & $P$ value \\
\hline \multicolumn{4}{|c|}{ Demographics } \\
\hline${ }^{*}$ Male sex $(\%)$ & $42(79,2 \%)$ & $33(73,3 \%)$ & 0,494 \\
\hline Age mean (year) & $64,0(60,8-67,1)$ & $67,4(63,3-71,2)$ & 0,135 \\
\hline $\operatorname{BMI}\left(\mathrm{kg} / \mathrm{m}^{\wedge} 2\right)$ & $28,1(26,7-29,4)$ & $28,8(27,2-30,5)$ & 0,708 \\
\hline \multicolumn{4}{|c|}{ Co-pathologies } \\
\hline${ }^{\delta}$ Charlson Comorbidity index & $3(2-4)$ & $3(1,5-5,5)$ & 0,218 \\
\hline Fypertension (\%) & $22(41,5 \%)$ & $26(57,8 \%)$ & 0,110 \\
\hline Diabetes (\%) & $11(20,8 \%)$ & $9(20 \%)$ & 0,927 \\
\hline${ }^{\ddagger}$ Obesity $(\%)$ & $15(28,3 \%)$ & $15(33,3 \%)$ & 0,592 \\
\hline F Cardiovascular Disease (\%) & $6(11,3 \%)$ & $12(26,7 \%)$ & 0,052 \\
\hline Respiratory Disease (\%) & $6(11,3 \%)$ & $10(22,2 \%)$ & 0,148 \\
\hline$\neq$ Oncological Disease (\%) & $3(5,7 \%)$ & $3(6,7 \%)$ & 0,837 \\
\hline \# Immunodepression (\%) & $6(11,3 \%)$ & 0 & $0,020^{*}$ \\
\hline \multicolumn{4}{|c|}{ Characteristics at admission } \\
\hline${ }^{\delta}$ Day of presentation since the start of symptoms & $7(5-10)$ & $7(3,5-9)$ & $0,047^{*}$ \\
\hline${ }^{\delta}$ Respiratory Rate & $25(21,5-31,5)$ & $23(20-26)$ & $0,045^{*}$ \\
\hline${ }^{8}$ PSI & $86(64,5-101,5)$ & $81(61,0-101,5)$ & 0,346 \\
\hline APACHE 2 & $10(7-14)$ & $9(6-11)$ & $0,040^{*}$ \\
\hline
\end{tabular}


Effect of Pronation and Postural Changes on Non-intubated Patients with Respiratory Failure Due to Covid-19 Interstitial Pneumonia

\begin{tabular}{|l|c|c|c|}
\hline $\mathrm{pH}$ & $7,47(7,45-7,48)$ & $7,45(7,43-7,46)$ & 0,050 \\
\hline $\mathrm{pCO} 2(\mathrm{mmHg})$ & $32,0(31,0-33,1)$ & $32,1(30,7-33,5)$ & 0,931 \\
\hline $\mathrm{P} / \mathrm{F}(\mathrm{mmHg})$ & $178,7(156,6-200,7)$ & $188,7(165,3-212,0)$ & 0,571 \\
\hline $\mathrm{SatO}_{2}(\%)$ & $91,5(90,1-93,0)$ & $91,3(90,2-92,4)$ & 0,439 \\
\hline $\mathrm{HcO}_{3}-(\mathrm{mmol} / \mathrm{l})$ & $22,9(22,1-23,7)$ & $22,5(21,4-23,7)$ & 0,574 \\
\hline Lactate $\left.^{\prime} \mathrm{mEq} / \mathrm{l}\right)$ & $1,6(1,4-1,9)$ & $1,9(1,3-2,6)$ & 0,291 \\
\hline${ }^{*}$ Necessity of FIO \\
\hline
\end{tabular}

Table 2: Comparison between patients mobilized in prone/lateral position and patients mobilized in sitting position.

Legend: All quantitative continuous data were reported as mean (standard deviations), quantitative ordinal variables (ర) were reported with median (interquartile range); all categorical variables ( $\ddagger$ ) were reported with frequencies (percentage). APACHE II (Acute Physiology and Chronic Health Evaluation II); BMI (Body Mass Index); DNI (Do Not Intubate); FIO 2 (Fraction of Inspired Oxygen); P/F $\left(\mathrm{PaO}_{2} / \mathrm{FIO}_{2}\right)$; PEEP (Positive End-Expiratory Pressure); PSI (Pneumonia Severity Index); $\mathrm{SpO}_{2}$ (peripheral capillary oxygen saturation). $* \mathrm{P}<0.05$.

In table 3, we enlisted the average times in position and $\mathrm{SpO}_{2}$ gain due to the mobilization. In figure 3 , we represented the distribution of $\mathrm{SpO}_{2}$ during the different mobilizations. Compared to the sitting position, prone and lateral positions were associated with higher improvement in mean $\mathrm{SpO}_{2}$ at fixed levels of $\mathrm{FIO}_{2}$. Notably, a partial benefit on $\mathrm{SpO}_{2}$ persisted for a short time after resupination, despite the short duration of the cycles. Regarding pronation, we documented two episodes of displacement of peripheral venous access, due to spontaneous resupination of the patients; no other complications were recorded.

\begin{tabular}{|l|c|c|c|c|}
\hline & $\boldsymbol{\Delta 1}$ & $\boldsymbol{\Delta 2}$ & $\boldsymbol{\Delta 3}$ & $\begin{array}{c}\text { Mean daily time } \\
\text { in decubitus }\end{array}$ \\
\hline Sitting position & +2.50 & $-0,75$ & +1.75 & 266 minutes \\
\hline Lateral position & +4.50 & -2.52 & +1.87 & 639 minutes \\
\hline Prone Position & $+5,25$ & $-1,60$ & +3.68 & 594 minutes \\
\hline
\end{tabular}

Table 3: $\mathrm{SpO}_{2}$ modification in different positions, at fixed $\mathrm{FIO}_{2}$.

Legend: $\Delta 1$ identifies the mean difference in $\mathrm{SpO}_{2}$ between supine

and sitting/lateral/prone position; $\Delta 2$ identifies the mean difference in $\mathrm{SpO}_{2}$ from before to after resupination; $\Delta 3$ identifies the mean difference in $\mathrm{Sp}_{2}$ in the supine position before and after the mobilization ( 1 hour after resupination). $\mathrm{FIO}_{2}$ (Fraction of Inspired Oxygen); $\mathrm{SpO}_{2}$ (peripheral capillary oxygen saturation).
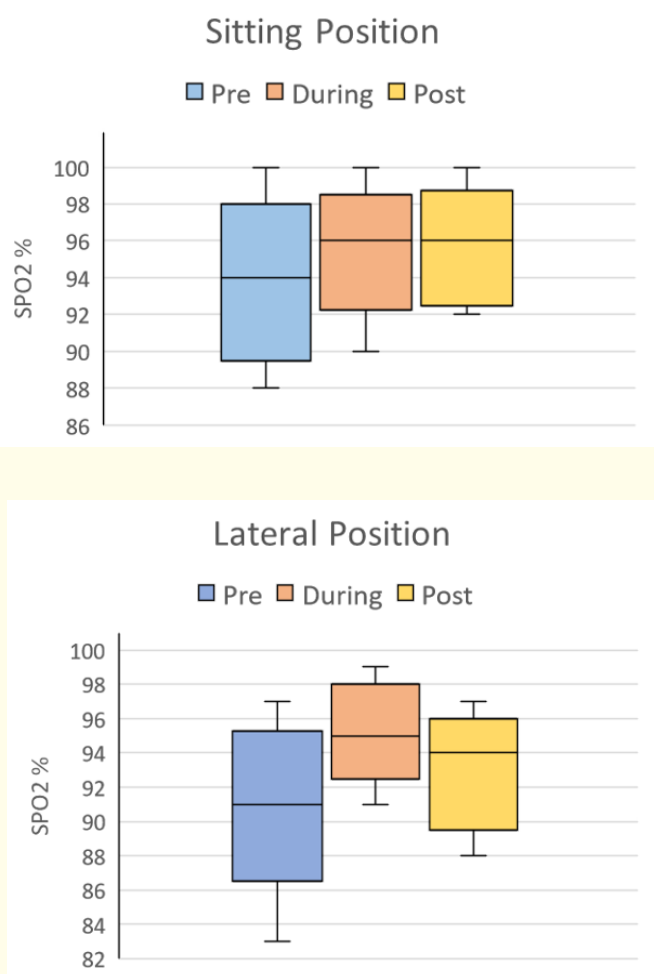

Citation: Lorenzo Porta., et al. "Effect of Pronation and Postural Changes on Non-intubated Patients with Respiratory Failure Due to Covid-19 Interstitial Pneumonia". Acta Scientific Medical Sciences 5.12 (2021): 94-103. 


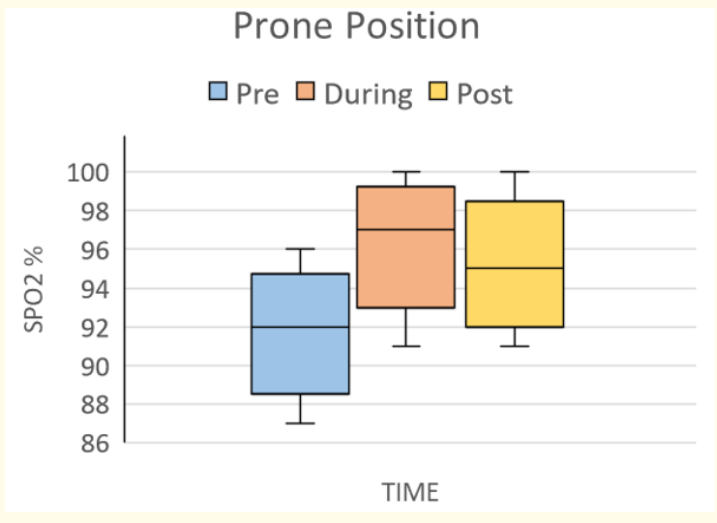

Figure 3: Box and Whiskers Plot on the distribution of Sp02 in different decubitus, before, during and after mobilization.

In table 4, we displayed the crude and adjusted odds ratios. The odds ratio with confidence interval (CI) for the need of intubation was 0,239 (CI95 0,102-0,556). After adjustment the odds ratio was 0,045 (CI95 0,008-0,244). The crude and adjusted odds ratio for intubation in the non-DNI population were 0,137(CI95 0,044-0,422) and after adjustment 0,076 (CI95 0,014-0,404). We calculated the crude odds ratio for mortality in the DNI subgroup, which resulted 0,338 (CI95 0,068-1,678, p 0,184), after adjustment we did not achieve statistical significance.

\begin{tabular}{|c|c|c|c|c|}
\hline & $\begin{array}{c}\text { Crude Odds Ratio } \\
\text { (OR, } \\
95 \% \\
\text { Confidence } \\
\text { Interval) }\end{array}$ & $\begin{array}{c}\mathbf{P} \\
\text { Value }\end{array}$ & $\begin{array}{c}\text { Confounder } \\
\text { adjusted } \\
\text { Odds Ratio } \\
\text { (OR, } \\
95 \% \\
\text { Confidence } \\
\text { Interval) }\end{array}$ & P Value \\
\hline $\begin{array}{l}\text { Necessity } \\
\text { of } \\
\text { intubation }\end{array}$ & $\begin{array}{c}0,239 \\
(0,102-0,556)\end{array}$ & 0,001 & $\begin{array}{c}0,045 \\
(0,008-0,244)\end{array}$ & $<0,001$ \\
\hline $\begin{array}{l}\text { Intubation } \\
\text { for non } \\
\text { DNI }\end{array}$ & $\begin{array}{c}0,137 \\
(0,044-0,422)\end{array}$ & 0,001 & $\begin{array}{c}0,076 \\
(0,014-0,404)\end{array}$ & 0,002 \\
\hline
\end{tabular}

Table 4: ODDs Ratio (ORs).

\section{Discussion}

The role of prone ventilation in hypoxemic ARF has already been thoroughly investigated and, despite some conflicting evidences, PP became a standard treatment for refractory hypoxia in
ARDS. In fact, several trials demonstrated that PP increases $\mathrm{PaO}_{2}$ in ARDS patients and, thus, reduces $\mathrm{FIO}_{2}$ requirements [17-19,27,28]. Furthermore, it has been demonstrated that this benefit lingers for hours after resupination and might improve each time prone ventilation is repeated $[20,21]$. However, due to important limitations of the studies, a definite conclusion whether PP could induce a mortality benefit has not been reached. In fact, while early randomized trials and meta-analyses reported no mortality benefit of PP for ARDS patients [22,23], the large PROSEVA study and several successive meta-analyses demonstrated that early, high-dose prone ventilation increased survival in patients with severe ARDS [26-28]. The PROSEVA trial observed a reduced 28-day and 90-day mortality due to PP ventilation, without excess risk of complications and with less need for rescue therapies. However, these conclusions could not be generalized because of important limitations of the study. In fact, not only the strict exclusion criteria allowed prone ventilation to be applied only to a selected minoritarian group of patients, but, despite randomization, patients' groups were not matched.

In 2015, Scaravilli., et al. applied PP to a different subset of patients. The group described for the first time the systematic application of PP in 15 awake, spontaneously breathing, non-intubated patients with hypoxemic ARF. Even though the study had several limitations (such as the retrospective design and the lack of a formal protocol) it evidenced an association between PP and a significant improvement in oxygenation, and suggested the use of PP as an alternative way to recruit the dorsal lung regions without relying on high-pressure ventilation [31]. Recently, Ding., et al. published a prospective observational cohort study analyzing the role of PP combined with ventilation with either high flow nasal cannula or NIV in awake non intubated patients with moderate to severe ARDS. Their data, collected from a sample of 20 patients mainly affected by viral pneumonia, suggested that early PP combined with HFNC/NIV might avoid intubation [36].

Similarly to the two aforementioned studies, we focused on a cohort of awake, spontaneously breathing patients and we decided to evaluate whether PP could be beneficial in non-intubated patients with ARF due to SARS-CoV-2 infection in an emergency/ medical ward. We found a strong association between both prone and lateral positions and avoidance of ETI. This benefit was not influence by any patients' characteristics that could have affected the prognosis of SARS-CoV-2 pneumonia (such as, gender, age, 
BMI, diabetes mellitus, hypertension, cardiovascular, respiratory or oncological disease, immunodepression, day of presentation to the emergency department, APACHE II, necessity of PEEP or $\mathrm{FIO}_{2}>50 \%$ at admission, DNI decision or treatment with Tocilizumab). The aforementioned beneficial effect could be related to the improvement in overall oxygenation in lateral and prone positions, as demonstrated by the improvement in mean $\mathrm{SpO}_{2}$. It must be noted that a small subgroup of our patients did not achieve any improvement in $\mathrm{SpO}_{2}$ due to pronation. This difference in response to lateral and prone position could have been due to the different phenotypes of the disease. In fact, as noted by Marini and Gattinoni $[37,38]$, from a theoretical and physiological point of view, this pathology displays two different respiratory phenotypes: one characterized by low elastance, low ventilation-perfusion (VA/Q) ratio, low lung weight and low lung recruitability (type L), and the other one (type H) characterized by opposite features (high elastance, high VA/Q ratio, high lung weight, high lung recruitability). While the latter shares similar features with classic ARDS and benefits from classic protective ventilation strategy, in the former type hypoxemia is due to the loss of hypoxic pulmonary vasoconstriction and blood flow autoregulation. Lacking the prerequisite for higher PEEP to work, the aim of NIV in type L patients is to redistribute pulmonary perfusion, improving the VA/Q relationship.

Our overall findings are consistent with both previous physiopathological and experimental studies; however, some differences with them must be considered. First, our population, composed of spontaneously breathing, non-intubated patients with mild to severe ARF, is more similar to the one usually found in medical departments, compared to an ICU population (our P/F ranged from 40 to $299 \mathrm{mmHg}$ ). In fact, all our patients were evaluated first by the intensivists in the emergency room before admission to the ward, in order to prevent ETI delay and all patients meeting the ETI criteria in the emergency room were admitted to the ICU directly. Second, borrowing the PP ventilation from an intensive care setting, we had to adapt it to a medical setting. In fact, having a partially autonomous population, able to change decubitus on his will, we opted for shorter cyclic periods of mobilization. This protocol allowed patients' relief while granting an average time in decubitus of 6-8 hour per day (an overall decubitus time comparable with Ding., et al. experience). Third, we decided to evaluate the necessity of ETI and not actual intubation. This decision was taken to include in the analysis patients considered DNI, which constitute a grow- ing subgroup population in time of scarcity of invasive ventilatory devices and bed in ICUs. For this reason, meeting the criteria for ETI did not imply actual intubation of the patient, in fact, due to the critical situation, this decision was left to collegial discussion with intensivists and was subjected to extra-ordinary circumstances. Last, even though PP ventilation was not routinely performed in our ward, we had no major complications, apart from two peripheral venous access losses due to autonomous resupination of the patients. Thus, we strongly support the feasibility of this practice in medical wards.

Some potential bias of our research must be noted. First, the observational retrospective design of the study could be viewed as a limitation, however, given the strong rationale of applying PP to ARF, the choice to prospectively randomize patients could have been debatable. Accordingly, only patients who categorically a priori refused prone or lateral decubitus fell in our comparison group. Second, compared to the sitting group, patients that accepted lateral and prone positions were mobilized during the night as well. This could have created a negative bias due to the different mobilization time and the different degree of attention required by the patient and provided by the medical staff. However, given the strong physio-pathological rationale and the different increase in the $\mathrm{SpO}_{2}$ registered in the different positions, the effect of this bias was probably minimal. Third, it must be taken into consideration that we used $\mathrm{SpO}_{2}$ as indirect estimator of $\mathrm{PaO}_{2}$ because it was easier to register and more used routinely in a medical ward (setting where our data can be applied), and because we were limited by the number of arterial blood gas analysis that we could performed in patients without arterial access. Fourth, among the different drug treatment prescribed during the hospitalization, we have only considered Tocilizumab as a possible confounder in the analysis. In fact, during the course of the study we updated the SARS-CoV-2 therapy according to the international evidences, in both groups at the same time. Thus, the magnitude of a hypothetical bias in the analysis due to different therapeutic schemes was negligible. Fifth, in the comparison between patients that were mobilized and not, the first appeared to be more severe with higher APACHE II, need for $\mathrm{FIO}_{2}>50 \%$ and prescription of Tocilizumab. To avoid potential positive bias, these characteristics were taken into consideration in the regression analysis. Sixth, our results might have been affected by the fact that during the hospitalization we changed the type of respiratory device, PEEP and $\mathrm{FIO}_{2}$ levels to maximize 
oxygenation. However, these changes were independent from the mobilization, applied to every patient and, thus, should have not severely influenced our data.

Despite the aforementioned limitations, we believe that our study, composed of one of the biggest cohorts of SARS-CoV-2 patients, could spur interest among other physicians. In fact, we claimed that the systematical application of either prone or lateral positions induce a substantial beneficial effect on $\mathrm{SpO}_{2}$ in awake, spontaneously breathing patients with ARF due to SARS-CoV-2 pneumonia. Furthermore, we examined and supported lateral positioning, which has never been fully studied before and which could be a valid option for patients not bearing prone positioning.

\section{Conclusion}

Our results support:

- The systematical use of prone and lateral positions to correct hypoxia, and thus reduce the need for ETI.

- The feasibility of this practice in medical wards, to save resources and counteract the possible scarcity of both ventilators and ICU beds.

Our subpopulation analysis demonstrated a benefit of prone and lateral position on ETI rate for the non-DNI population, but no statistical evidence of a mortality benefit in the DNI population.

\section{Acknowledgment}

We would like to ackowledge the work of: Albertini Valentina, Battistini Marta, Bettoni Giuseppe, Bergamaschi Marta, Bossi Ilaria, Capsoni Nicolò, Didomenico Sandro, Ferrazzi Davide, Fumagalli Roberto, Galli Claudia, Ghezzi Roberto, Guarnieri Linda, Licata Giada, Periti Enrica, Strozzi Marco, Tombini Valeria.

\section{Bibliography}

1. Guan WJ., et al. "Clinical characteristics of 2019 novel coronavirus infection in China”. New England Journal of Medicine 382 (2020): 1708-1720.

2. Shi H., et al. "Radiological findings from 81 patients with COVID-19 pneumonia in Wuhan, China: a descriptive study". Lancet Infectious Diseases 20 (2020): 425.

3. Zhao W., et al. "Relation Between Chest CT Findings and Clinical Conditions of Coronavirus Disease (COVID-19) Pneumonia: A Multicenter Study". The American Journal of Roentgenology (2020): 1.
4. Wang D., et al. "Clinical Characteristics of 138 Hospitalized Patients With 2019 Novel Coronavirus-Infected Pneumonia in Wuhan, China". The Journal of the American Medical Association 323.11 (2020): 1061-1069.

5. Chen N., et al. "Epidemiological and clinical characteristics of 99 cases of 2019 novel coronavirus pneumonia in Wuhan, China: a descriptive study". Lancet 395 (2020): 507-513.

6. Huang C., et al. "Clinical features of patients infected with 2019 novel coronavirus in Wuhan, China”. Lancet 395 (2020): 497506.

7. Wu C., et al. "Risk Factors Associated with Acute Respiratory Distress Syndrome and Death in Patients with Coronavirus Disease 2019 Pneumonia in Wuhan, China". The Journal of the American Medical Association Internal Medicine 180.7 (2020): 934-943.

8. Guérin C., et al. "Prone positioning in severe acute respiratory distress syndrome". The New England Journal of Medicine 368 (2013): 2159-68.

9. Pan C., et al. "Lung Recruitability in SARS-CoV-2 Associated Acute Respiratory Distress Syndrome: A Single-center, Observational Study". American Journal of Respiratory and Critical Care Medicine (2020).

10. Alhazzani W., et al. "Surviving Sepsis Campaign: guidelines on the management of critically ill adults with Coronavirus Disease 2019 (COVID-19)". Intensive Care Medicine 46(2020):854887.

11. Lazzeri M., et al. "Respiratory physiotherapy in patients with COVID-19 infection in acute setting: a Position Paper of the Italian Association of Respiratory Physiotherapists (ARIR)". Monaldi Archives for Chest Disease 90.1 (2020).

12. Gattinoni L., et al. "Prone positioning in acute respiratory failure". In: Tobin MJ editor(s). Principles and Practice of Mechanical Ventilation. 2nd Edition. New York: McGraw-Hill Education (2006): 1081-1092.

13. Guerin C. "Ventilation in the prone position in patients with acute lung injury/acute respiratory distress syndrome". Current Opinion in Critical Care 12 (2006): 50-54.

14. Graf J and Marini JJ. "Do airway secretions play an underappreciated role in acute respiratory distress syndrome?" Current Opinion in Critical Care 14 (2008): 44-49.

15. Marini JJ. "Can we prevent the spread of focal lung inflammation”? Critical Care Medicine 38 (2010): 574-581. 
16. Gattinoni L., et al. "Stress and strain within the lung". Current Opinion in Critical Care 18.1 (2012): 42-47.

17. Gattinoni L., et al. "Prone position in acute respiratory distress syndrome: rationale, indications, and limits". American Journal of Respiratory Critical Care Medicine 188.11 (2013): 12861293.

18. Mentzelopoulos SD., et al. "Prone position reduces lung stress in severe acute respiratory distress syndrome". European Respiratory Journal 25 (2005): 534-544.

19. Pelosi P., et al. "Prone position in acute respiratory distress syndrome”. European Respiratory Journal 20 (2020): 1017.

20. Chatte G., et al. "Prone position in mechanically ventilated patients with severe acute respiratory failure". American Journal of Respiratory and Critical Care Medicine 155 (1997): 473.

21. Fridrich P., et al. "The effects of long-term prone positioning in patients with trauma-induced adult respiratory distress syndrome”. Anesthesia and Analgesia 83 (1996): 1206.

22. Taccone P., et al. "Prone positioning in patients with moderate and severe acute respiratory distress syndrome: a randomized controlled trial". The Journal of the American Medical Association 302 (2009): 1977.

23. Guerin C., et al. "Effects of systematic prone positioning in hypoxemic acute respiratory failure: a randomized controlled trial". The Journal of the American Medical Association 292 (2004): 2379.

24. Gattinoni L., et al. "Effect of prone positioning on the survival of patients with acute respiratory failure". The New England Journal of Medicine 345 (2001): 568.

25. Mancebo J., et al. "A multicenter trial of prolonged prone ventilation in severe acute respiratory distress syndrome". American Journal of Respiratory and Critical Care Medicine 173 (2006): 1233.

26. Lee JM., et al. "The efficacy and safety of prone positional ventilation in acute respiratory distress syndrome" updated study-level meta-analysis of 11 randomized controlled trials. Critical Care Medicine 42 (2014): 1252.

27. Bloomfield R., et al. "Prone position for acute respiratory failure in adults". Cochrane Database Systematic Reviews (2015): CD008095.

28. Munshi L., et al. "Prone Position for Acute Respiratory Distress Syndrome: A Systematic Review and Meta-Analysis". Annals of the American Thoracic Society 14 (2017): S280.
29. A.Valter C., et al. "Response to the prone position in spontaneously breathing patients with hypoxemic respiratory failure". Acta Anaesthesiologica Scandinavica 47 (2003): 416-8.

30. Feltracco P., et al. "Non-invasive ventilation in prone position for refractory hypoxemia after bilateral lung transplantation". Clinical Transplant 23 (2009): 748-50.

31. Scaravilli V., et al. "Prone positioning improves oxygenation in spontaneously breathing nonintubated patients with hypoxemic acute respiratory failure". A retrospective study, Journal of Critical Care (2015): 1390-1394.

32. Grasselli G., et al. "Critical Care Utilization for the COVID-19 Outbreak in Lombardy, Italy: Early Experience and Forecast During an Emergency Response". The Journal of the American Medical Association (2020).

33. Ferrer M., et al. "Noninvasive ventilation in severe hypoxemic respiratory failure: a randomized clinical trial”. American Journal of Respiratory and Critical Care Medicine 168 (2003): 1438-1444.

34. Plant PK., et al. "Early use of non-invasive ventilation for acute exacerbations of chronic obstructive pulmonary disease on general respiratory wards: a multicentre randomised controlled trial". Lancet 355 (2000): 1931-1935.

35. Brambilla AM., et al. "Helmet CPAP vs. oxygen therapy in severe hypoxemic respiratory failure due to pneumonia" [published correction appears in Intensive Care Medicine. Intensive Care Medicine 40.7 (2014): 942-949.

36. Ding, L., et al. "Efficacy and safety of early prone positioning combined with HFNC or NIV in moderate to severe ARDS: a multi-center prospective cohort study". Critical Care 24 (2020): 28.

37. Marini JJ and Gattinoni L. "Management of COVID-19 Respiratory Distress". The Journal of the American Medical Association (2020).

38. Gattinoni L., et al. "COVID-19 pneumonia: different respiratory treatments for different phenotypes?" Intensive Care Medicine 46.6 (2020): 1099-1102.

\section{Volume 5 Issue 12 December 2021 (C) All rights are reserved by Lorenzo Porta., et al.}

